\title{
A PRELIMINARY TEST OF THE CALIBRATION OF THE HB SYSTEM IN TERMS OF ABSOLUTE MAGNITUDES AND INTRINSIC COLOURS FOR EARLY-TYPE STARS
}

\author{
A. F. J. MOFFAT, Th. SCHMIDT-KALER, and N. VOGT \\ Astronomisches Institut der Ruhr-Universität Bochum, F.R.G.
}

\begin{abstract}
The dependency of the $\mathrm{H} \beta$-index on two physical parameters is briefly discussed. The previous calibration of $M_{v}(\beta)$ (Fernie, 1965 or Crawford, 1971) is confirmed for main sequence $O$ and B stars; however, the $\beta$-values of the later B-supergiants tend to be too large for their absolute magnitudes. The problem of filtering out stars with marginal emission is emphasized.
\end{abstract}

The existing calibrations of the $\mathrm{H} \beta$-index as a function of absolute magnitude $M_{v}$ (Graham, 1967; Fernie, 1965; Crawford, 1971) neglect the fact that $\beta$ depends on at least two parameters; e.g. temperature and pressure. In addition, certain areas of the Hertzsprung-Russell-diagram show discrepancies; for example the $\beta$-values of A-type supergiants are too large for their absolute magnitudes (see, e.g., Figure 1).

The fundamental objective of $\mathrm{H} \beta$-measurements is the determination of distances especially of field stars with spectral types $\mathrm{O}, \mathrm{B}, \mathrm{A}$. For this purpose photometry in at least three colours is also necessary (e.g. $U B V$ ). Since the relations

$$
\begin{array}{lrl}
\beta \text {-index } \quad \beta & =f_{1}\left(M_{v}, T_{\text {eff }}\right), \\
\text { intrinsic colour } C_{0} & =f_{2}\left(M_{v}, T_{\text {eff }}\right)
\end{array}
$$

hold, it is clear that the final calibration must be carried out simultaneously in terms of the photometric quantities $U B V$ and $\beta$ if the spectral type of the star is not known. Since the first equation has two solutions of $M_{v}$ for a given $\beta$ it is necessary to distinguish between $\mathrm{O}-\mathrm{B} 9$ and A0-F stars. Also stars with shells show emission lines, and must be considered separately. A calibration system which satisfies the above conditions is in preparation.

Here we wish only to present a preliminary test of Crawford's (1971) calibration $M_{v}(\beta)$. Fernie's (1965) calibration is practically equivalent to Crawford's. For this we have taken observations of stars in the following young open clusters: the nuclei of $h$ and $\chi$ Persei (Crawford, Glaspey and Perry, 1970), III Cep (Crawford and Barnes, 1970), I Sco (Crawford et al., 1971) and our observations of 60 stars in 30 southern clusters. This large number of clusters has the advantage that systematic errors which may arise in individual cases are randomized. In all cases the distance moduli have been determined independently from $\mathrm{H} \beta$ using $U B V$ photometry. For $\mathrm{h}$ and $\chi$ Persei, III Cep and I Sco, the values $V_{0}-M_{v}=11.7,9.3$ and 11.5, respectively, have been used; for the 30 southern clusters the distance moduli have been derived by Moffat and Vogt (1973) and Vogt and Moffat $(1972,1973)$. All data have been reduced to $R=A_{v} / E_{B-V}=3.2$ for $\mathrm{A} 0$-stars. The internal errors of our $\mathrm{H} \beta$-values are \pm 0 m.008 for $V<10^{\mathrm{m}}$; external errors are less than 0.005 . The results are presented in Figure 1. 


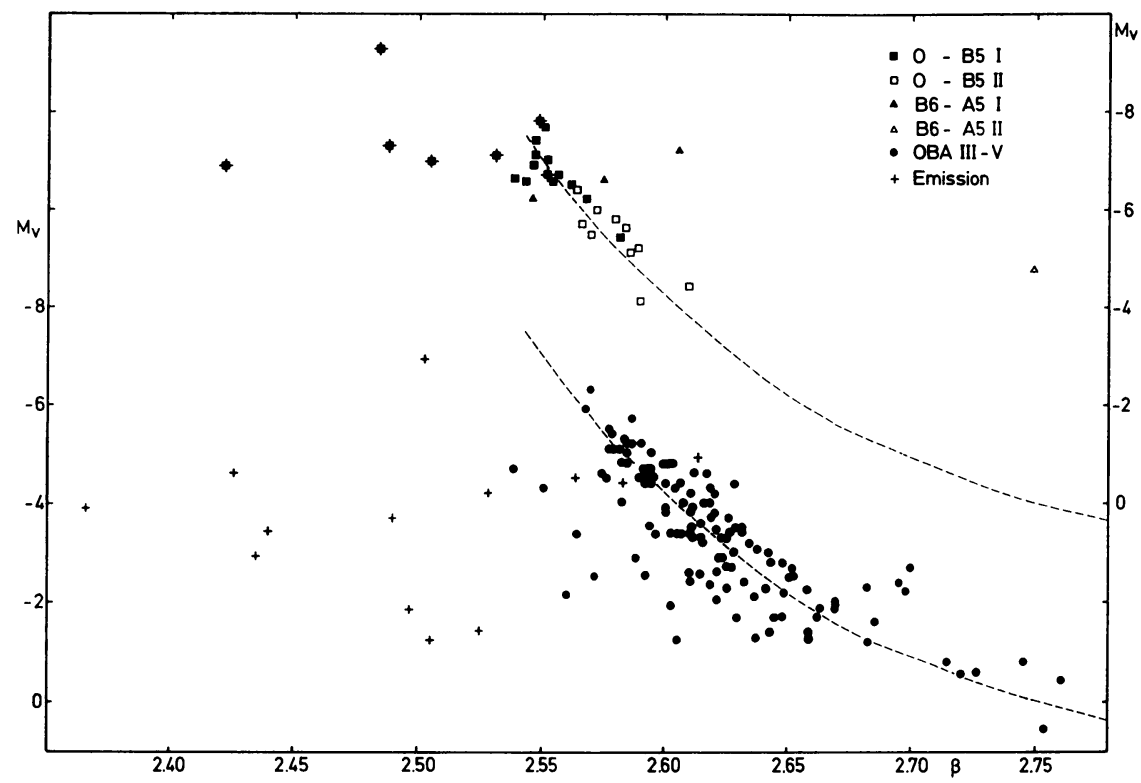

Fig. 1. The calibration of the visual absolute magnitude $M_{v}$ as a function of the $\beta$-index. The diagram is separated into two parts: supergiants and class III-V stars. The dotted curves represent the calibration of Crawford (1971).

The known emission B-stars (shown by crosses) occupy the region to the left of the calibration curve, as expected. The frequency distribution about the calibration curve suggests two components: a symmetrical component centered on the curve, and an asymmetrical component corresponding to the emission stars. Therefore, in computing the dispersion we have omitted nine stars in addition to the spectroscopically known emission stars. The resulting scatter is

$\begin{array}{llll}\text { Range } & \text { Spectraltype } & \sigma_{M_{v}} & \text { Number } \\ \beta<2.61 & & & \\ \beta \leqslant 2.63 & \text { O-B5 I-II } & \pm 0.53 & 22 \\ 2.63<\beta<2.68 & \text { O-B1 III-V } & \pm 0.58 & 82 \\ & \text { B2-5 III-V } & \pm 0.54 & 27\end{array}$

Supergiant emission B-stars tend to be 0.5 brighter than non-emission stars of the same spectral type. Taking into account the errors of the distance moduli and the interstellar absorption, the scatter is reduced to 0.4 . This value includes the effects of observational errors, rotation and spectroscopic binaries.

We confirm Crawford's and Fernie's $M_{v}(\beta)$ calibrations. Stars with $\beta<2.54$ are certainly emission objects; emission stars between this line and the calibration curve clearly present a problem when they are field OB-stars in case no sufficient spectroscopic information is available. 


\section{References}

Crawford, D. L., Glaspey, J. W., and Perry, C. L.: 1970, Astron. J. 75, 822.

Crawford, D. L. and Barnes, J. V.: 1970, Astron. J. 75, 946.

Crawford, D. L., Barnes, J. V., Hill, G., and Perry, C. L.: 1971, Astron. J. 76, 1048.

Crawford, D. L.: 1971, private communication.

Fernie, J. D.: 1965, Astron. J. 70, 575.

Graham, J. A.: 1967, Monthly Notices Roy. Astron. Soc. 135, 377.

Moffat, A. F. J. and Vogt, N.: 1973, Astron. Astrophys. Suppl., in press.

Vogt, N. and Moffat, A. F. J.: 1972, Astron. Astrophys. Suppl. 7, 133.

Vogt, N. and Moffat, A. F. J.: 1973, Astron. Astrophys. Suppl. 9, 97.

\section{DISCUSSION}

Schild: Your value for net scatter in the absolute magnitude calibration of \pm 0.4 is strikingly small; from the statistics of double stars, have you been able to assess the contribution to this scatter caused by spectroscopic binaries?

Schmidt-Kaler: We did not correct for binaries. As to their percentage I might quote Petrie's result that around half of the early type B stars are binaries. A large proportion of the companions would, however, be too faint to affect appreciably the calibration. On the other hand we do not want to correct for binaries since we wish to apply the results to very distant and very faint clusters.

Crampton: It wasn't clear to me whether your results are based only on the three northern clusters or on more extensive data?

Schmidt-Kaler: We have used the MK-classified stars only of the clusters observed by Crawford et al. as a skeleton, the rest is from 30 southern open clusters which had thus far never been observed photometrically. 